\title{
Integrated Management of Major Foliar and Soil-Borne Diseases of Peanut (Arachis hypogaea L.) with Fungicides, Trichoderma and Castor Cake
}

\author{
K.S. Jadon ${ }^{1 *}$, P.P. Thirumalaisamy ${ }^{2}$, Vinod Kumar ${ }^{3}$, V.G. Koradia ${ }^{2}$ and R.D. Padavi \\ ${ }^{1}$ ICAR-Central Arid Zone Research Institute, Jodhpur - 342003 (Rajasthan), India \\ ${ }^{2}$ ICAR-Directorate of Groundnut Research, IvnagarRoad, PB. No. 5, \\ Junagadh - 362001 (Gujarat), India \\ ${ }^{3}$ ICAR-National Research Centre on Litchi, Mushahari, Muzaffarpur- 842002 (Bihar), India \\ *Corresponding author
}

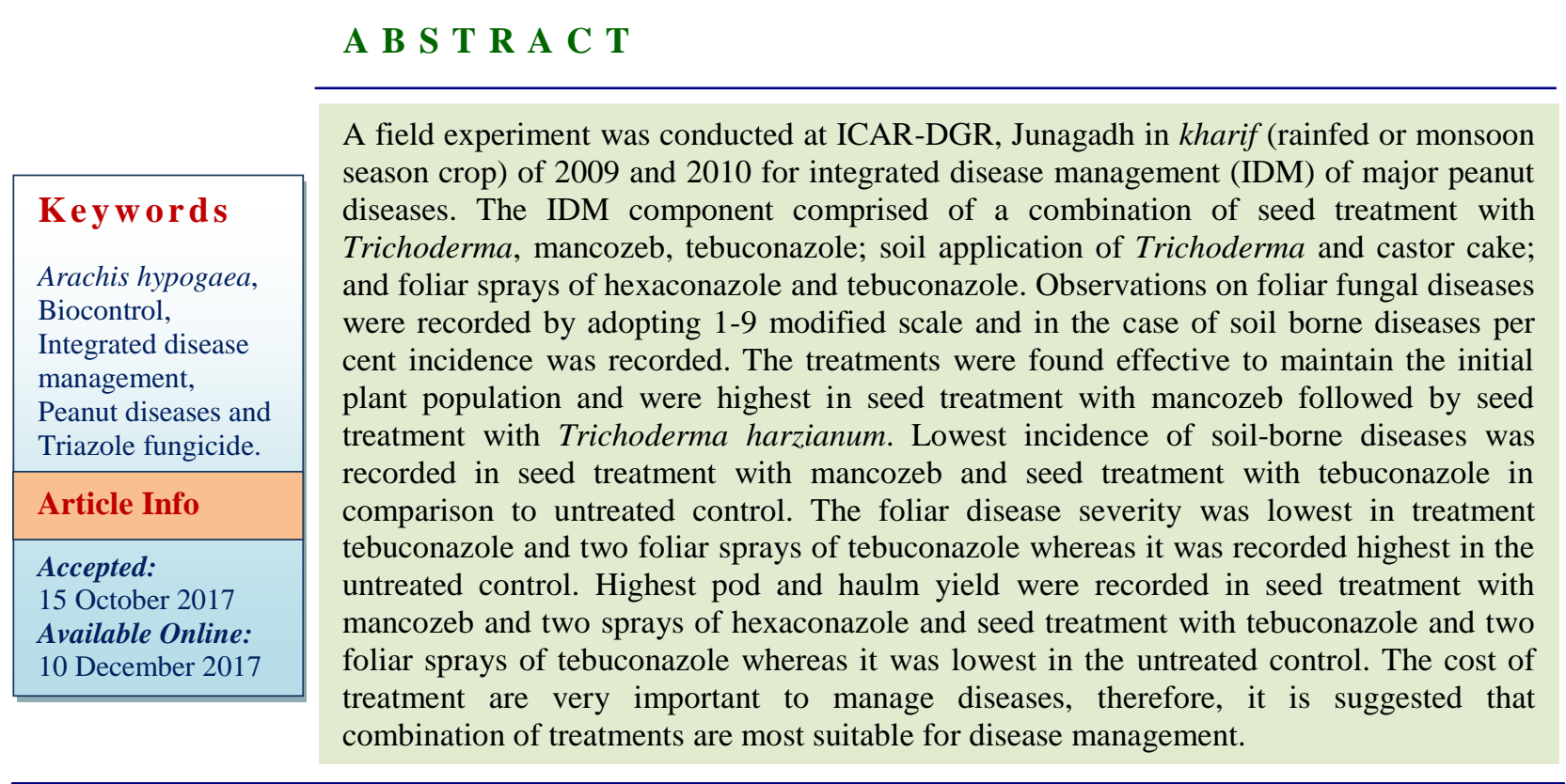

\section{Introduction}

Peanut or groundnut (Arachis hypogaea L.) is an important crop with high levels of proteins, carbohydrates, vitamins and minerals contained within seeds (Moss and Rao, 1995). While the cultivation of peanut may occur over a wide range of climatic conditions, soil type, temperature, and amount of rainfall and its distribution during crop season need special attention. Well-drained, sandy soils are best suited for peanut production (Beasley et al., 1997). In 2013, the world peanut production was 45.7 million tonne from 25.4 million ha area with an average productivity of $1796.2 \mathrm{~kg} \mathrm{ha}^{-1}$ (FAO, 2013).

In India, peanut is an important oilseed crop, contributing about 36 per cent of the total oilseed production. India is the largest grower and second largest producer of peanut in the world. It is cultivated as Kharif (rainfed or 
monsoon season crop) and Rabi-summer (irrigated, sown in winter and harvested in the spring) crop and the major peanut growing states are Gujarat, Andhra Pradesh, Tamil Nadu, Karnataka, Rajasthan, and Maharashtra which contributes around $90 \%$ of area and production. It is cultivated in 5.5 million ha area, divided in Kharif (4.6) and Rabi (1.0) with a production of about 9.7 million tonne (Kharif- 7.8 and Rabi-1.9) and productivity $1750 \mathrm{~kg} \mathrm{ha}^{-1}$. The average yield of Rabipeanut is around $1929 \mathrm{~kg} \mathrm{ha}^{-1}$, whereas Kharif-peanut is around $1712 \mathrm{kgha}^{-1}$ which is lower than major peanut growing countries (GOI, 2014). This may be attributed to the rainfed-cultivation of this crop coupled with damage caused by several pests and diseases. Among foliar fungal diseases, only a few are economically important such as early and late leaf spots commonly called as 'tikka' disease and rust. Early leaf spot (ELS) is caused by Cercosporaarachidicola Hori (Perfect stageMycosphaerella arachidis Deighton and late leaf spot (LLS) is caused by Phaeois ariopsispersonata (Berk \& Curt) V. Arx (Perfect stage - Mycosphaerella berkeleyi Jenkins). Rust is caused by Puccinia arachidis Speg. Among seed and soil-borne diseases, collar rot also called crown rot or seedling blight (Aspergillus niger Van Tieghem, A. pulverulentus (McAlp) Thom), stem rotor Sclerotium wilt (Sclerotium rolfsii Sacc), dry wilt or dry root rot (Macrophomina phaseolina (Tassi) Goid= Rhizoctonia bataticola (Taub.) Butler) have been recognized as major diseases. Economically important virus diseases are peanutbud necrosis disease (PBND), peanut stem necrosis disease (PSND), peanut mottle and peanut clump. Peanut bud necrosis is sap transmissible and vectored by Thripspalmi Karny. Peanut mottle is transmitted in a non-persistent manner by aphids, Aphis craccivora and Myzus persicae. In India, among foliar fungal diseases, ELS, LLS and rust are economically important which are widely distributed and can cause yield losses up to $70 \%$. Seed and soilborne diseases cause severe seedling mortality resulting in the patchy crop, stand mostly in sandy loam soils and reduce yields from 25 to $50 \%$. PBND has been reported to cause yield losses up to $50 \%$ in the early infected crop. Besides reducing pod yield, it also affects the fodder quality of haulm (DGR, 2014). However, the incidence or severity of the disease may vary from season to season.

The magnitude of losses due to diseases is high all over the world. In general, the disease management programme involves the most use of pesticides and very rarely farmers go for other alternatives such as cultural, physical, biological methods etc. Hence, comprehensive pest management programme is most desirable. The management of diseases of peanut through a single biological or chemical method is very difficult and impractical. Various control measures need to be suitably integrated to cover a broad spectrum of pests. The approach, therefore, should be an integrated protection of the crop rather than integrated management of individual pest. There are a few research efforts where the pest control measures have been integrated and there appears a scope for further improvement in integrated pest management in peanut. Integration of best disease management practices for peanut diseases would be an effective approach and also the need of the hour. Appropriate use of fungicides with bio-control agents could be a cost-effective and eco-friendly approach. In the present studies, Trichoderma harzianum and some recommended fungicides were used for management of major soil borne and foliar fungal diseases of peanut.

\section{Materials and Methods}

The experiment was conducted in randomized block design with four replications in $15 \mathrm{~m}^{2}$ $(5 \mathrm{~m} \times 3 \mathrm{~m})$ plot size with $30 \mathrm{~cm} \times 10 \mathrm{~cm}$ spacing at experimental farm of ICAR- 
Directorate of Groundnut Research (DGR), Junagadh (N 2131', E 70³6', $60 \mathrm{~m}$ asl), Gujarat (India) during the kharif season in 2009 and 2010 using peanut cultivar GG-20 and GG-2, respectively. The climate of the region is semi-arid with a mean annual rainfall of $850 \mathrm{~mm}$. The rainfall is mainly confined during June to September. The monthly average temperature is minimum in January $\left(12{ }^{\circ} \mathrm{C}\right)$ and maximum in May (42 $\left.{ }^{\circ} \mathrm{C}\right)$. The soils of the experimental field are clayey, mixed, hyperthermic, lithic haplustepts. These soils sometimes develop shallow cracks and are very dark grey, slightly calcareous, moderately alkaline, clayey texture underlain by weathered or hard miliolitic limestone. They are also strongly alkaline, strongly stony, clayey soils.

The soils are shallow and the depth ranges from $25-50 \mathrm{~cm}$. The soils are moderately well drained and are moderately permeable. They have medium runoff and are moderately eroded. The clay texture imparts good nutrient reserve capacity and good water holding capacity. Physicochemical parameters and nutrient status of experimental plotsup to a depth of $0-15 \mathrm{~cm}$ were the organic carbon about $0.69 \%$, EC $0.14 \mathrm{dSm}^{-1}, \mathrm{pH} 8.3$, bulk density $1.20 \mathrm{~g} \mathrm{~cm}^{-3}$, water retention 28.8 at $1 / 3$ bar and 15.4 at 15 bar, exchangeable cations (Ca 37.8, Mg 9.9, Na 1.7, K 0.3 CEC $53.2 \mathrm{cmol} \mathrm{kg}^{-1}$ soil), ESP $3.2 ; \mathrm{CaCO}_{3} 3.0 \%$, available $\mathrm{N} 72.1 \mathrm{~kg} \mathrm{ha}^{-1}, \mathrm{P} 17.3 \mathrm{~kg} \mathrm{ha}^{-1}, \mathrm{~K}$ $176.6 \mathrm{~kg} \mathrm{ha}^{-1}$, Fe $8.74 \mathrm{mg} \mathrm{kg}^{-1}$, Mn $9.28 \mathrm{mg}$ $\mathrm{kg}^{-1}$, $\mathrm{Zn} 1.5 \mathrm{mg} \mathrm{kg}^{-1}$ and $\mathrm{Cu} 1.42 \mathrm{mg} \mathrm{kg}^{-1}$.In spite of limited depth, soils are responsive to balanced fertilizer application particularly the inclusion of $\mathrm{N}, \mathrm{P}, \mathrm{K}, \mathrm{Zn}$ and $\mathrm{Fe}$ in the fertilizer schedule. (Singh et.al., 1998)

Bioagent, T. harzianum (NRCG, T-170) maintained at Plant Pathology section of DGR were used in the experiment. Mass multiplication of $T$. harzianum was done on the castor cake which was also a treatment.
For this Trichoderma culture was multiplied on sorghum grains for seven days. This was thoroughly mixed in castor cake seven days before field application. The moisture level and humidity were maintained by covering with gunny bags.

To ensure sufficient disease incidence of collar rot and stem rot, inoculums of Aspergillus niger@ $25 \mathrm{~kg} \mathrm{ha}^{-1}$ and Sclerotium rolfsii@100 $\mathrm{kg} \mathrm{ha}^{-1}$, multiplied on sorghum grain was added to soils before sowing and at 20 days after sowing (DAS), respectively. A spray of fungicides was scheduled as $1^{\text {st }}$ spray at the first appearance of symptoms of foliar diseases followed by $2^{\text {nd }}$ spray after 15 days.

Observations on foliar fungal diseases were recorded by adopting1-9 modified scale (Subrahmanyam et al., 1995) and in the case of soil borne diseases per centincidence was computed. Per cent Soil-borne Disease incidence, Per cent efficacy of disease control (PEDC), Per cent yield increase over disease control (PIDC) was calculated by using the following formulae:

Per cent Soil-borne Disease incidence $=$ (number of infected plant units /total number of plant units assessed) $\mathrm{x} 100$

Per cent efficacy over Disease Control $($ PEDC $)=[($ Disease severity or incidence in control- Disease severity or incidence in treatment)/ Disease severity or incidence in control] x 100

Per cent yield increase over Disease Control $(\mathrm{PIDC})=[($ Yield in treatments-Yield in Control)/Yield in Control] x100

The incremental cost benefit ratio (ICBR) and Benefit: Cost Ratio (B: C ratio) of all the treatments was calculated using following formulae: 
$\mathrm{ICBR}=\mathrm{ICBR}=$ Additional income received (from the particular treatment) / Additional cost incurred (for the particular treatment)

B: C ratio $=$ Income received (from the particular treatment) / Cost incurred (for the particular treatment)

The data were analysed following standard statistical procedures using DSAASTAT software (Onobri, 2007) and analysis of variance (ANOVA) was worked out. Data was subjected to $F$ test and least significant difference (LSD) between means was calculated at $5 \%$ significance level $(\mathrm{p}<0.05)$.

\section{Results and Discussion}

The results of the experiments indicated that all the treatments significantly reduced the incidence of soil-bornediseases and severity of foliar fungal diseases over the untreated control. All the treatments were found effective to maintain the initial plant population (numbers) in the field (Fig. 1). Significantly higher plant population was recorded in seed treatment with mancozeb (273) followed by seed treatment with $T$. harzianum (260) as compared to control (238). Lowest incidence of soil-borne diseases viz., aflaroot (Fig. 2), collar rot (Fig. 3 ) and stem rot (Fig. 4) were recorded during both the years of seed treatment with mancozeb and two spray of hexaconazole $\left(\mathrm{T}_{2}\right)$ (1.1, 8.8, and 11.6per cent, respectively) followed by seed treatment with tebuconazole $\left(1.5 \mathrm{gkg}^{-1}\right.$ seed) and two foliar spray of tebuconazole $\left(\mathrm{T}_{3}\right) \quad(0.9, \quad 9.0$ and 11.9, respectively) in comparison to untreated control (1.7, 14.3 and 23.8, respectively) and significantly reduced the disease incidence (Table 3).

The highest PEDC (per cent efficiency over disease control) was observed in $\mathrm{T}_{2}$ and $\mathrm{T}_{3}$ for aflaroot (35.3 and 47.1, respectively), collar rot (38.5 and 37.1, respectively) and stem rot
(51.3 and 50.0, respectively) in comparison to other treatments applied (Table 1).

The observation on foliar diseases revealed that all the treatments had significantly reduced PDI (per cent disease incidence) in comparison to untreated control (Table 2). Seed treatment with tebuconazole and two foliar sprays of tebuconazole $\left(T_{3}\right)$ was found most effective among all the treatments. The lowest PDI of ELS (Fig. 5), LLS (Fig. 6) and rust (Fig. 7) (5.9, 3.7 and 4.2, respectively) were recorded in $\mathrm{T}_{3}$ whereas it was recorded highest (7.2, 6.8 and 6.3, respectively) in the untreated control. The PEDC (per cent efficacy over disease control) was also very high in $\mathrm{T}_{3}$ in comparison to other treatments for ELS (17.4), LLS (45.7) and rust (33.8) (Table 1).

The data on biological yield (pod and haulm) of peanut showed the significant effect of treatments on the enhancement of the yield over the untreated control (Table 4). The highest pod yield was recorded in $\mathrm{T}_{2}$ and $\mathrm{T}_{3}$ (1637 kg/ha and $1627 \mathrm{~kg} / \mathrm{ha}$, respectively) whereas it was lowest in untreated control (1425 kg/ha) (Fig. 8). The haulm yield was also found highest in $\mathrm{T}_{2}(3115 \mathrm{~kg} / \mathrm{ha})$ and $\mathrm{T}_{3}$ $(3097 \mathrm{~kg} / \mathrm{ha})$ in comparison to control $(2743$ $\mathrm{kg} / \mathrm{ha}$ ) (Fig. 8). Both the treatment $\mathrm{T}_{2}$ (14.9) and $\mathrm{T}_{3}$ (14.2) showed the highest PIDC (per cent increase over disease control) for pod yield and for haulm yield, respectively, among all the treatments (Table 1). The incremental cost benefit ratio (ICBR) calculated on the basis of prevailing market price indicated that it was highest in $\mathrm{T}_{1}$ (1:8.3), $\mathrm{T}_{2}$ (1:8.7) and $\mathrm{T}_{3}(1: 4.9)$. The data on ICBR showed that the cost of treatment was very important for management decision. Based on the result, it is suggested that treatment $\mathrm{T}_{2}$-seed treatment with mancozeb (3 $\left.\mathrm{g} \mathrm{kg}^{-1}\right)$ and two sprays of hexaconazole $(1 \mathrm{ml}$ $\left.\mathrm{l}^{-1}\right)$ will be most economical for the management of peanut diseases. 
The following treatments (fungicides) were used as seed dressing

\begin{tabular}{|c|c|}
\hline Treatment & Treatment Detail \\
\hline $\mathrm{T}_{1}$ & $\begin{array}{l}\text { Seed treatment with } T \text {. harzianum }\left(10 \mathrm{~g} \mathrm{~kg}^{-1} \text { seed }\right) \text { and two sprays of } \\
\text { hexaconazole } 5 \% \mathrm{SC}\left(1 \mathrm{ml} \mathrm{^{-1 }}\right)\end{array}$ \\
\hline $\mathrm{T}_{2}$ & $\begin{array}{l}\text { Seed treatment with mancozeb75\% WP }\left(3 \mathrm{~g} \mathrm{~kg}^{-1}\right) \text { and two sprays of } \\
\text { hexaconazole } 5 \% \mathrm{SC}\left(1 \mathrm{ml} \mathrm{l}^{-1}\right)\end{array}$ \\
\hline $\mathrm{T}_{3}$ & $\begin{array}{l}\text { Seed treatment with tebuconazole } 2 \text { DS }\left(1.5 \mathrm{~g} \mathrm{~kg}^{-1} \text { seed }\right) \text { and two foliar sprays } \\
\text { of tebuconazole } 25.9 \% \mathrm{EC}\left(1 \mathrm{ml} \mathrm{l}^{-1}\right)\end{array}$ \\
\hline $\mathrm{T}_{4}$ & $\begin{array}{l}\text { Soil application of } T \text {. harzianum }\left(4.0 \mathrm{~kg} \mathrm{ha}^{-1}\right) \text { and castor cake }\left(250 \mathrm{~kg} \mathrm{ha}^{-1}\right) \\
\text { and two sprays of hexaconazole } 5 \% \mathrm{SC}\left(1 \mathrm{ml} \mathrm{l}^{-1}\right)\end{array}$ \\
\hline $\mathrm{T}_{5}$ & Seed treatment with $T$. harzianum $\left(10 \mathrm{~g} \mathrm{~kg}^{-1}\right)+\mathrm{T}_{4}$ \\
\hline $\mathrm{T}_{6}$ & trol (without any seed treatment, only water spray) \\
\hline
\end{tabular}

The detail of fungicides used are given below

\begin{tabular}{|l|l|l|l|}
\hline Common name & Trade name & Chemical name & Manufacturing company \\
\hline $\begin{array}{l}\text { hexaconazole/ } \\
\text { Contaf Plus }\end{array}$ & Hexaconazole 5\% SC & $\begin{array}{l}\text { 2-(2,4-Dichlorophenyl)-1-(1H-1,2,4-triazol-1- } \\
\text { yl)hexan-2-ol }\end{array}$ & $\begin{array}{l}\text { Rallis India } \\
\text { Mumbai }\end{array}$ \\
\hline Tebuconazole & $\begin{array}{l}\text { Folicur250 EC } \\
(25.9 \% \text { w/w) }\end{array}$ & $\begin{array}{l}( \pm)-1-(4-C h l o r o p h e n y l)-4,4-d i m e t h y l-3-(1 \mathrm{H}, \\
1,2,4-\text { triazol-1-ylmethyl)pentan-3-ol }\end{array}$ & Bayer CropScience Ltd. \\
\hline Tebuconazole & Raxil 2DS & $\begin{array}{l}\text { (RS)- 1-(4-Chlorophenyl)- 4,4-dimethyl-3-(1H, } \\
1,2,4-t \text { triazol-1-ylmethyl)pentan- 3-ol }\end{array}$ & Bayer CropScience Ltd. \\
\hline Mancozeb & $\begin{array}{l}\text { TATA M-45 } \\
\text { (mancozeb 75\% WP) }\end{array}$ & $\begin{array}{l}\text { zinc;manganese(2+);N-[2- } \\
\text { (sulfidocarbothioylamino)ethyl]carbamodithioate }\end{array}$ & $\begin{array}{l}\text { Rallis India Limited, } \\
\text { Mumbai }\end{array}$ \\
\hline
\end{tabular}

Table.1 Effect of different treatment on plant population and percentefficacy over disease control of major soil-borne and foliar diseases of peanut

\begin{tabular}{|c|c|c|c|c|c|c|c|c|c|c|}
\hline \multirow{2}{*}{ Treatment } & \multirow{2}{*}{$\begin{array}{l}\text { Initial plant } \\
\text { population }\end{array}$} & \multicolumn{3}{|c|}{$\begin{array}{l}\text { Per cent Efficacy over Disease } \\
\text { Control }\end{array}$} & \multicolumn{3}{|c|}{$\begin{array}{l}\text { Per cent Efficacy Over } \\
\text { Disease Control }\end{array}$} & \multirow{2}{*}{$\begin{array}{l}\text { Per cent } \\
\text { Yield } \\
\text { Increase } \\
\text { Over } \\
\text { Disease } \\
\text { Control } \\
\end{array}$} & \multirow[t]{2}{*}{$\begin{array}{l}\text { B: C } \\
\text { Ratio }\end{array}$} & \multirow[t]{2}{*}{ ICBR } \\
\hline & & Aflaroot & $\begin{array}{l}\text { Collar } \\
\text { rot }\end{array}$ & $\begin{array}{l}\text { Stem } \\
\text { rot }\end{array}$ & ELS & LLS & Rust & & & \\
\hline $\mathrm{T}_{1}$ & 260 & 23.5 & 32.9 & 50.4 & 10.5 & 34.0 & 29.9 & 9.6 & 0.68 & $1: 8.3$ \\
\hline $\mathrm{T}_{2}$ & 273 & 35.3 & 38.5 & 51.3 & 12.2 & 34.6 & 31.3 & 14.9 & 0.77 & $1: 8.7$ \\
\hline $\mathrm{T}_{3}$ & 242 & 47.1 & 37.1 & 50.0 & 17.4 & 45.7 & 33.8 & 14.2 & 0.74 & $1: 4.9$ \\
\hline $\mathrm{T}_{4}$ & 250 & 17.6 & 31.5 & 46.6 & 14.0 & 35.7 & 34.4 & 12.9 & 0.67 & $1: 2.4$ \\
\hline $\mathrm{T}_{5}$ & 246 & 11.8 & 30.1 & 46.2 & 11.1 & 39.5 & 29.1 & 8.1 & 0.62 & $1: 1.9$ \\
\hline $\mathrm{T}_{6}$ & 238 & 0.0 & 0.0 & 0.0 & 0.0 & 0.0 & 0.0 & 0.0 & 0.53 & - \\
\hline SEm \pm & \multirow{2}{*}{\multicolumn{10}{|c|}{$\begin{array}{l}7.36 \\
21.26\end{array}$}} \\
\hline LSD $<0.05$ & & & & & & & & & & \\
\hline \multicolumn{11}{|c|}{ 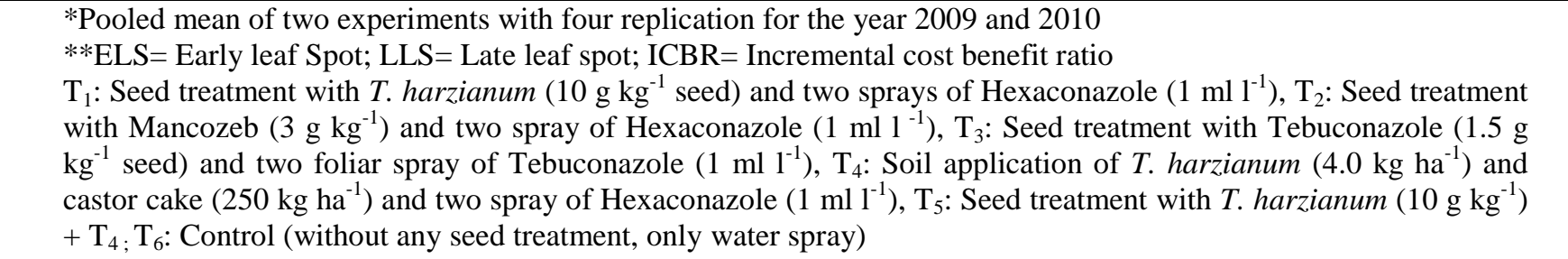 } \\
\hline
\end{tabular}


Table.2 Analysis of variance table for effects of different treatments on severity of early leaf spot, late leaf spot, and rust (2009, 2010 and Two Year Means)

\begin{tabular}{|c|c|c|c|c|c|c|c|c|c|c|}
\hline \multirow[b]{2}{*}{ Source of variation } & \multirow[b]{2}{*}{$d f^{x}$} & \multicolumn{3}{|c|}{ Early leaf spot } & \multicolumn{3}{|c|}{ Late leaf spot } & \multicolumn{3}{|l|}{ Rust } \\
\hline & & $\mathbf{M S}^{\mathbf{y}}$ & $F^{z}$ & $\mathbf{P}^{\mathbf{z}}$ & $\mathbf{M S}^{\mathbf{y}}$ & $F^{z}$ & $\mathbf{P}^{\mathbf{z}}$ & $\mathbf{M S}^{\mathbf{y}}$ & $F^{z}$ & $\mathbf{P}^{\mathbf{z}}$ \\
\hline \multicolumn{11}{|l|}{2009} \\
\hline Replication & 3.0 & 0.4 & 3.9 & & 0.3 & 2.8 & & 0.6 & 1.3 & \\
\hline Treatments & 5.0 & 0.9 & 8.8 & $<0.01$ & 0.5 & 4.0 & $<0.05$ & 1.1 & 2.1 & 0.1 \\
\hline Residual & 15.0 & 0.1 & & & 0.1 & & & 0.5 & & \\
\hline \multicolumn{11}{|l|}{2010} \\
\hline Replication & 3.0 & 0.0 & 0.1 & & 0.6 & 1.5 & & 1.2 & 6.8 & \\
\hline Treatments & 5.0 & 0.6 & 5.0 & $<0.01$ & 0.7 & 1.7 & 0.2 & 0.5 & 2.7 & 0.1 \\
\hline Residual & 15.0 & 0.1 & & & 0.4 & & & 0.2 & & \\
\hline \multicolumn{11}{|l|}{ Two Year Means } \\
\hline Replication & 3.0 & 0.1 & 4.4 & & 0.0 & 0.3 & & 0.3 & 1.0 & \\
\hline Treatments & 5.0 & 0.7 & 26.7 & $<0.01$ & 0.6 & 3.8 & $<0.05$ & 0.7 & 2.8 & 0.1 \\
\hline Residual & 15.0 & 0.0 & & & 0.1 & & & 0.3 & & \\
\hline
\end{tabular}

${ }^{\mathrm{X}}$ Degree of freedom; ${ }^{\mathrm{Y}}$ Mean square; ${ }^{\mathrm{Z}} \mathrm{F}$ value and probability of a greater F-value

Table.3 Analysis of variance table for effects of different treatments on incidence of afla root, collar rot and stem rot (2009, 2010 and Two Year Means)

\begin{tabular}{|c|c|c|c|c|c|c|c|c|c|c|}
\hline \multirow[b]{2}{*}{ Source of variation } & \multirow[b]{2}{*}{$d f^{x}$} & \multicolumn{3}{|c|}{ Aflaroot (\%) } & \multicolumn{3}{|c|}{ Collar rot $(\%)$} & \multicolumn{3}{|c|}{ Stem rot $(\%)$} \\
\hline & & $\mathbf{M S}^{\mathbf{y}}$ & $F^{z}$ & $\mathbf{P}^{\mathbf{z}}$ & $\mathbf{M S}^{\mathbf{y}}$ & $F^{z}$ & $\mathbf{P}^{\mathbf{z}}$ & $\mathbf{M S}^{\mathbf{y}}$ & $F^{z}$ & $\mathbf{P}^{\mathbf{z}}$ \\
\hline \multicolumn{11}{|l|}{2009} \\
\hline Replication & 3.0 & 0.2 & 0.6 & & 1.2 & 1.2 & & 1.4 & 2.1 & \\
\hline Treatments & 5.0 & 12.9 & 33.6 & $<0.01$ & 12.7 & 12.5 & $<0.01$ & 25.4 & 36.5 & $<0.01$ \\
\hline Residual & 15.0 & 0.4 & & & 1.0 & & & 0.7 & & \\
\hline \multicolumn{11}{|l|}{2010} \\
\hline Replication & 3.0 & 3.2 & 6.6 & & 0.5 & 0.3 & & 0.5 & 0.5 & \\
\hline Treatments & 5.0 & 9.9 & 20.3 & $<0.01$ & 9.4 & 5.6 & $<0.01$ & 5.5 & 5.3 & $<0.01$ \\
\hline Residual & 15.0 & 0.5 & & & 1.7 & & & 1.0 & & \\
\hline \multicolumn{11}{|l|}{ Two Year Means } \\
\hline Replication & 3.0 & 1.1 & 15.9 & & 0.1 & 0.6 & & 0.0 & 0.4 & \\
\hline Treatments & 5.0 & 8.0 & 110.5 & $<0.01$ & 2.4 & 16.4 & $<0.01$ & 3.2 & 33.5 & $<0.01$ \\
\hline Residual & 15.0 & 0.1 & & & 0.1 & & & 0.1 & & \\
\hline
\end{tabular}


Table.4 Analysis of variance table for effects of different treatments on peanut yield (2009, 2010 and Two Year Means)

\begin{tabular}{|c|c|c|c|c|c|c|c|}
\hline \multirow[b]{2}{*}{ Source of variation } & \multirow[b]{2}{*}{$d f^{x}$} & \multicolumn{3}{|c|}{ Pod Yield $\left(\mathrm{kg} \mathrm{ha}^{-1}\right)$} & \multicolumn{3}{|c|}{ Haulm Yield $\left(\mathrm{kg} \mathrm{ha}^{-1}\right)$} \\
\hline & & $\mathbf{M S}^{\mathbf{y}}$ & $F^{z}$ & $\mathbf{P}^{\mathbf{Z}}$ & $\mathbf{M S}^{\mathbf{y}}$ & $F^{z}$ & $\mathbf{P}^{\mathbf{z}}$ \\
\hline \multicolumn{8}{|l|}{2009} \\
\hline Replication & 3.0 & 49289 & 5.3 & & 150948 & 5.3 & \\
\hline Treatments & 5.0 & 11463 & 1.2 & 0.3 & 35106 & 1.2 & 0.3 \\
\hline Residual & 15.0 & 9320 & & & 28541 & & \\
\hline \multicolumn{8}{|l|}{2010} \\
\hline Replication & 3.0 & 48073 & 7.2 & & 147224 & 7.2 & \\
\hline Treatments & 5.0 & 56433 & 8.5 & 0.0 & 172827 & 8.5 & 0.0 \\
\hline Residual & 15.0 & 6666 & & & 20414 & & \\
\hline \multicolumn{8}{|l|}{ Two Year Means } \\
\hline Replication & 3.0 & 36794 & 6.3 & & 112682 & 6.3 & \\
\hline Treatments & 5.0 & 23755 & 4.1 & 0.0 & 72749 & 4.1 & 0.0 \\
\hline Residual & 15.0 & 5802 & & & 17769 & & \\
\hline
\end{tabular}

Fig.1 Effect of different treatments on initial plant population of Peanut. Least significant difference (LSD) values were calculated at $P<0.05$

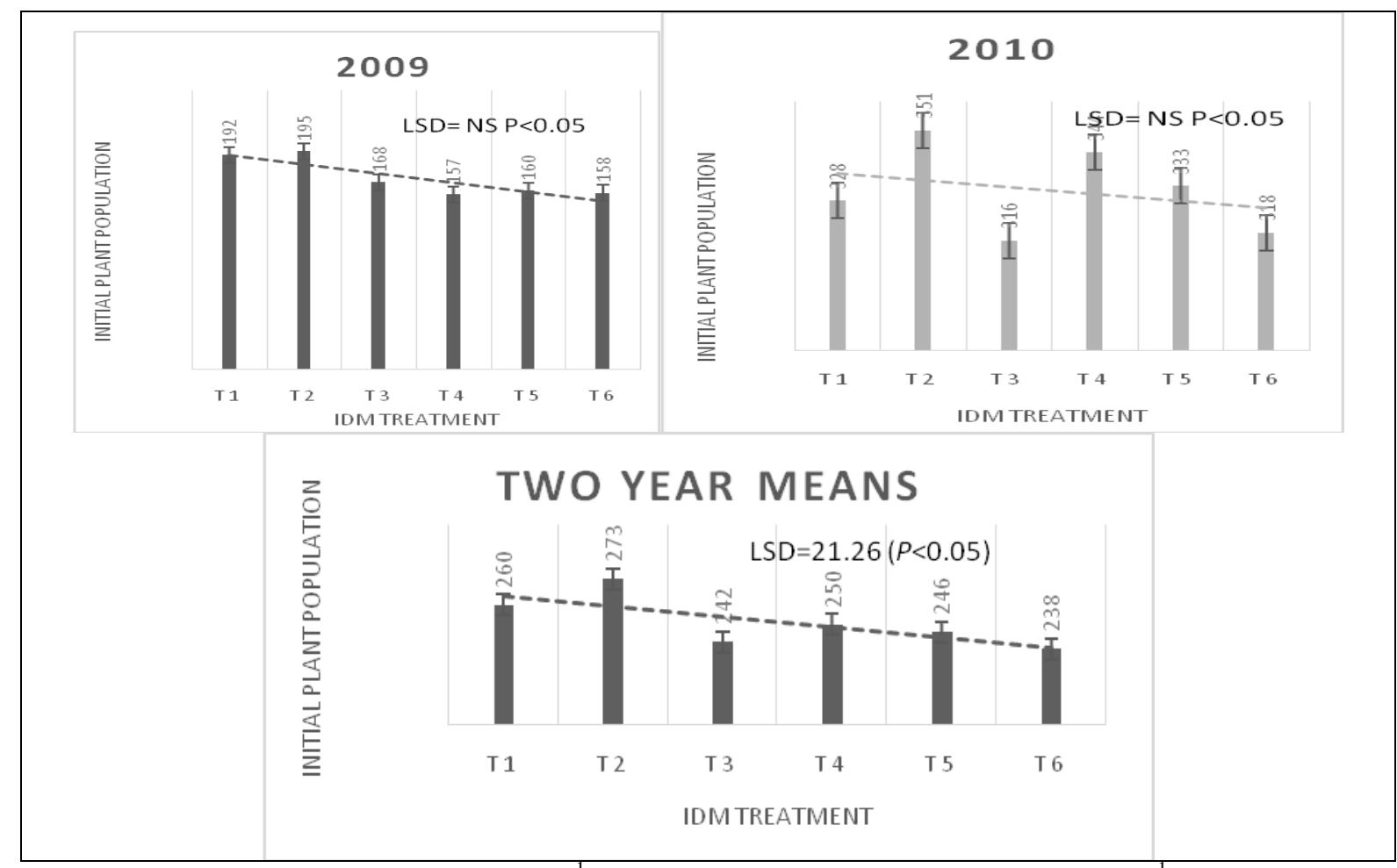

$\mathrm{T}_{1}$ : Seed treatment with T. harzianum $\left(10 \mathrm{~g} \mathrm{~kg}^{-1}\right.$ seed) and two sprays of hexaconazole $\left(1 \mathrm{ml} \mathrm{l}^{-1}\right), \mathrm{T}_{2}:$ Seed treatment with mancozeb $\left(3 \mathrm{~g} \mathrm{~kg}^{-1}\right)$ and two spray of hexaconazole $\left(1 \mathrm{ml} \mathrm{l}^{-1}\right), \mathrm{T}_{3}$ : Seed treatment with tebuconazole $\left(1.5 \mathrm{~g} \mathrm{~kg}^{-1}\right.$ seed) and two foliar spray of tebuconazole $\left(1 \mathrm{ml} \mathrm{l}^{-1}\right), \mathrm{T}_{4}$ : Soil application of T. harzianum $\left(4.0 \mathrm{~kg} \mathrm{ha}^{-1}\right)$ and castor cake $\left(250 \mathrm{~kg} \mathrm{ha}^{-1}\right)$ and two spray of hexaconazole $\left(1 \mathrm{ml} \mathrm{l}^{-1}\right), \mathrm{T}_{5}$ : Seed treatment with T. harzianum $\left(10 \mathrm{~g} \mathrm{~kg}^{-1}\right)+\mathrm{T}_{4}$; $\mathrm{T}_{6}$ : Control (without any seed treatment, only water spray) 
Fig.2 Effect of different treatments on aflaroot incidence. Least significant difference (LSD) values were calculated at $P<0.05$

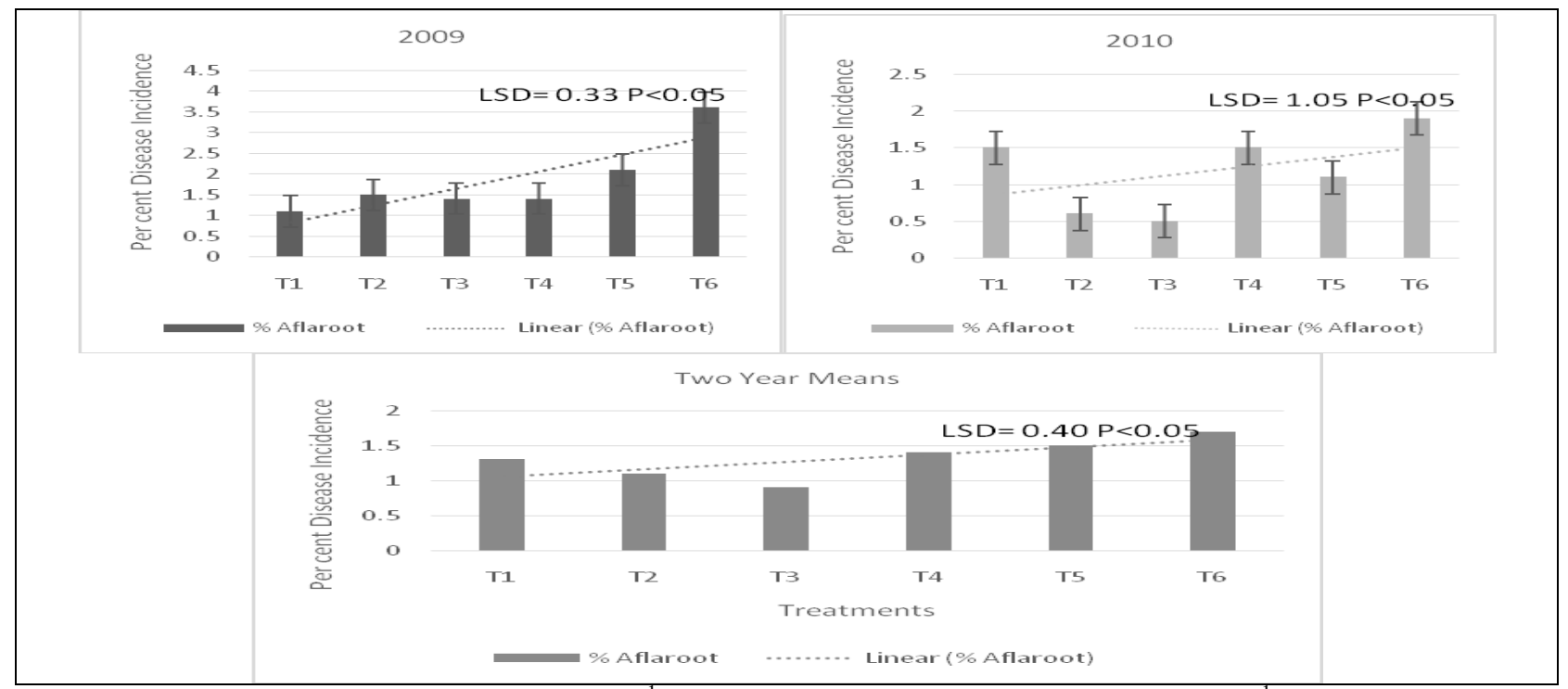

$\mathrm{T}_{1}$ : Seed treatment with $T$. harzianum $\left(10 \mathrm{~g} \mathrm{~kg}^{-1}\right.$ seed) and two sprays of hexaconazole $\left(1 \mathrm{ml} \mathrm{l}^{-1}\right), \mathrm{T}_{2}$ : Seed treatment with mancozeb $\left(3 \mathrm{~g} \mathrm{~kg}^{-1}\right)$ and two spray of hexaconazole $\left(1 \mathrm{ml} \mathrm{l}^{-1}\right), \mathrm{T}_{3}$ : Seed treatment with tebuconazole $\left(1.5 \mathrm{~g} \mathrm{~kg}^{-1}\right.$ seed) and two foliar spray of tebuconazole $\left(1 \mathrm{ml} \mathrm{l}^{-1}\right), \mathrm{T}_{4}$ : Soil application of T. harzianum $\left(4.0 \mathrm{~kg} \mathrm{ha}^{-1}\right)$ and castor cake $\left(250 \mathrm{~kg} \mathrm{ha}^{-1}\right)$ and two spray of hexaconazole $\left(1 \mathrm{ml} \mathrm{l}^{-1}\right), \mathrm{T}_{5}$ : Seed treatment with $T$. harzianum $\left(10 \mathrm{~g} \mathrm{~kg}^{-1}\right)+\mathrm{T}_{4}$; $\mathrm{T}_{6}$ : Control (without any seed treatment, only water spray)

Fig.3 Effect of different treatments on collar rot incidence. Least significant difference (LSD) values were calculated at $P<0.05$

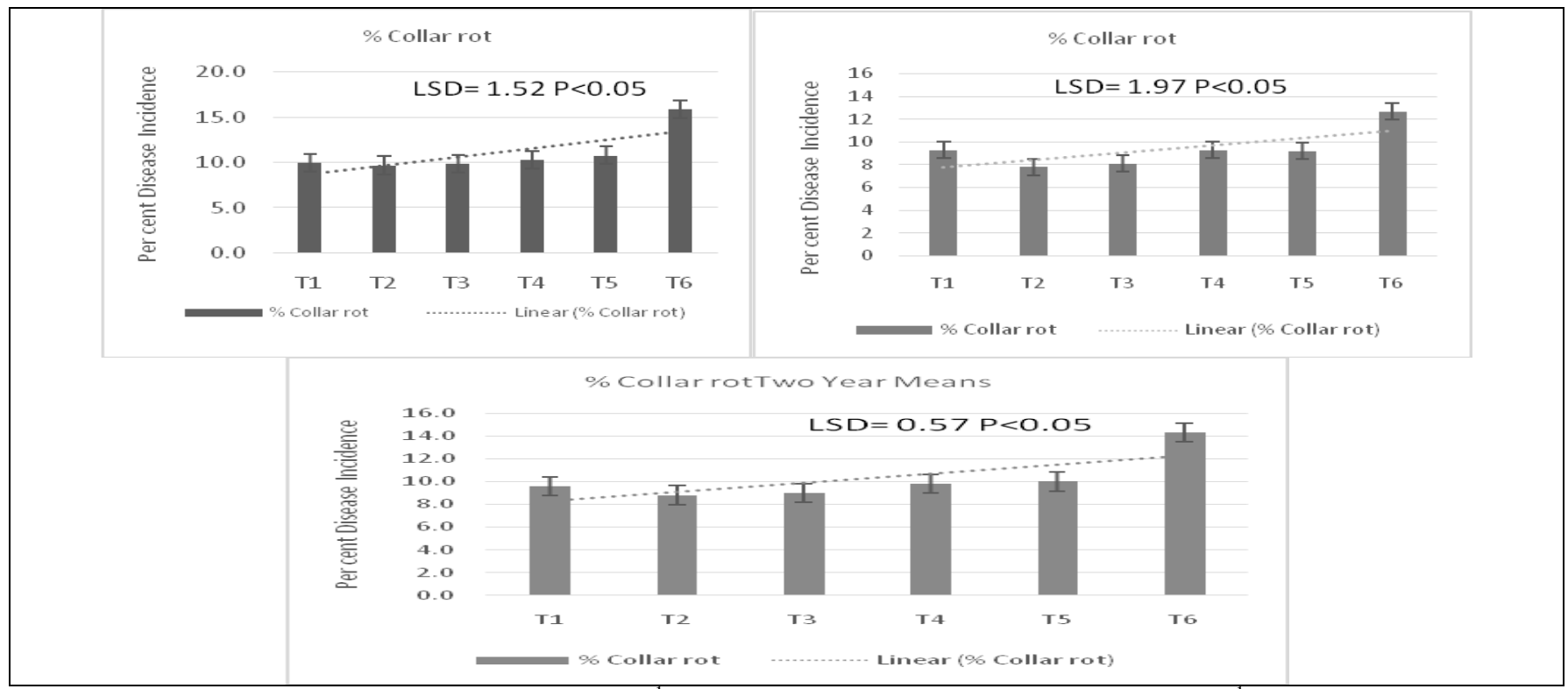

$\mathrm{T}_{1}$ : Seed treatment with $T$. harzianum $\left(10 \mathrm{~g} \mathrm{~kg}^{-1}\right.$ seed) and two sprays of hexaconazole $\left(1 \mathrm{ml} \mathrm{l}^{-1}\right), \mathrm{T}_{2}$ : Seed treatment with mancozeb $\left(3 \mathrm{~g} \mathrm{~kg}^{-1}\right)$ and two spray of hexaconazole $\left(1 \mathrm{ml} \mathrm{l}^{-1}\right), \mathrm{T}_{3}$ : Seed treatment with tebuconazole $\left(1.5 \mathrm{~g} \mathrm{~kg}^{-1}\right.$ seed) and two foliar spray of tebuconazole $\left(1 \mathrm{ml} \mathrm{l}^{-1}\right), \mathrm{T}_{4}$ : Soil application of T. harzianum $\left(4.0 \mathrm{~kg} \mathrm{ha}^{-1}\right)$ and castor cake $\left(250 \mathrm{~kg} \mathrm{ha}^{-1}\right)$ and two spray of hexaconazole $\left(1 \mathrm{ml} \mathrm{l}^{-1}\right), \mathrm{T}_{5}$ : Seed treatment with $T$. harzianum $\left(10 \mathrm{~g} \mathrm{~kg}^{-1}\right)+\mathrm{T}_{4}$; $\mathrm{T}_{6}$ : Control (without any seed treatment, only water spray) 
Fig.4 Effect of different treatments on stem rot incidence. Least significant difference (LSD) values were calculated at $P<0.05$

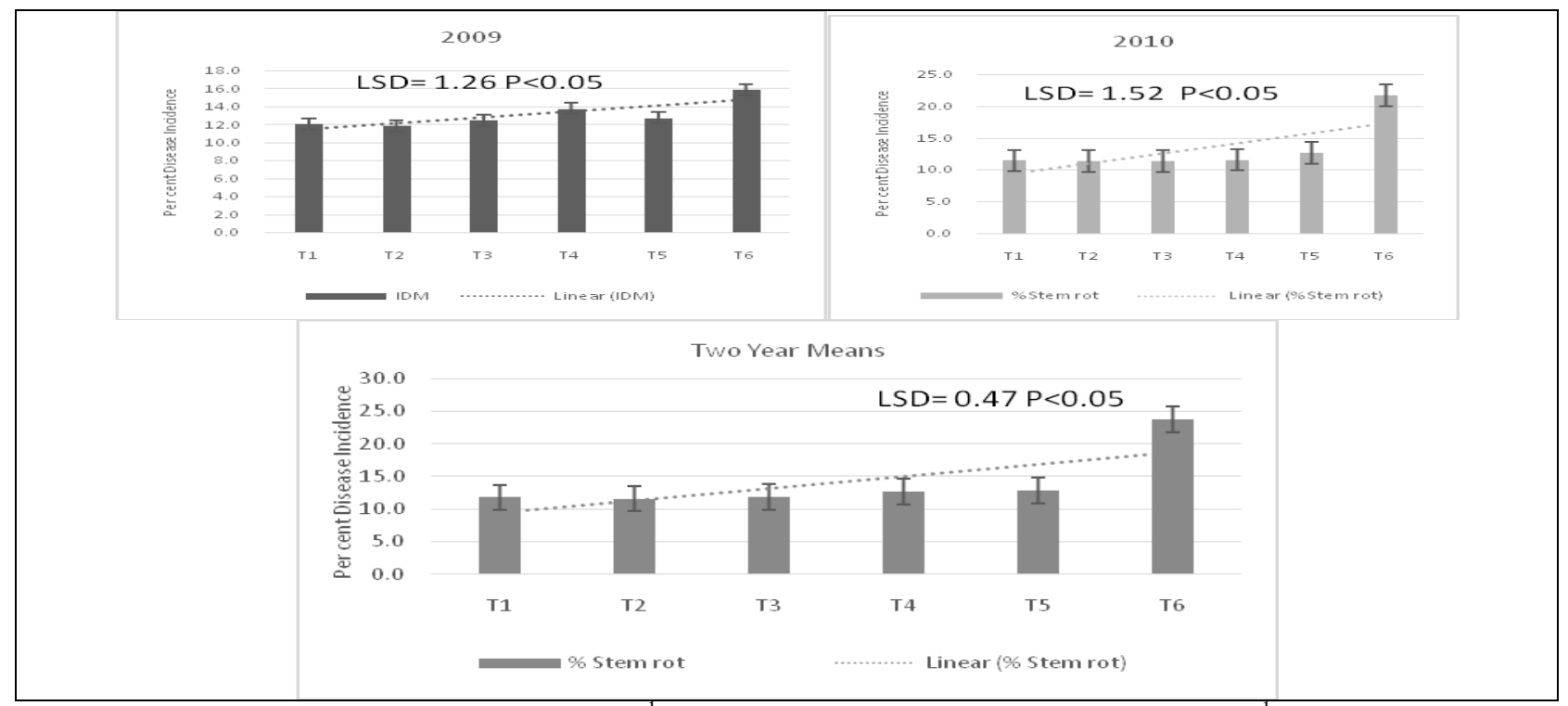

$\mathrm{T}_{1}$ : Seed treatment with $T$. harzianum $\left(10 \mathrm{~g} \mathrm{~kg}^{-1}\right.$ seed $)$ and two sprays of hexaconazole $\left(1 \mathrm{ml} \mathrm{l}^{-1}\right), \mathrm{T}_{2}$ : Seed treatment with mancozeb $\left(3 \mathrm{~g} \mathrm{~kg}^{-1}\right)$ and two spray of hexaconazole $\left(1 \mathrm{ml} \mathrm{l}^{-1}\right), \mathrm{T}_{3}$ : Seed treatment with tebuconazole $\left(1.5 \mathrm{~g} \mathrm{~kg}^{-1}\right.$ seed) and two foliar spray of tebuconazole $\left(1 \mathrm{ml} \mathrm{l}^{-1}\right), \mathrm{T}_{4}$ : Soil application of T. harzianum $\left(4.0 \mathrm{~kg} \mathrm{ha}^{-1}\right)$ and castor cake $\left(250 \mathrm{~kg} \mathrm{ha}^{-1}\right)$ and two spray of hexaconazole $\left(1 \mathrm{ml} \mathrm{l}^{-1}\right), \mathrm{T}_{5}$ : Seed treatment with $T$. harzianum $\left(10 \mathrm{~g} \mathrm{~kg}^{-1}\right)+\mathrm{T}_{4}$; $\mathrm{T}_{6}$ : Control (without any seed treatment, only water spray)

Fig.5 Effect of different treatments on early leaf spot (ELS) severity. Least significant difference (LSD) values were calculated at $P<0.05$

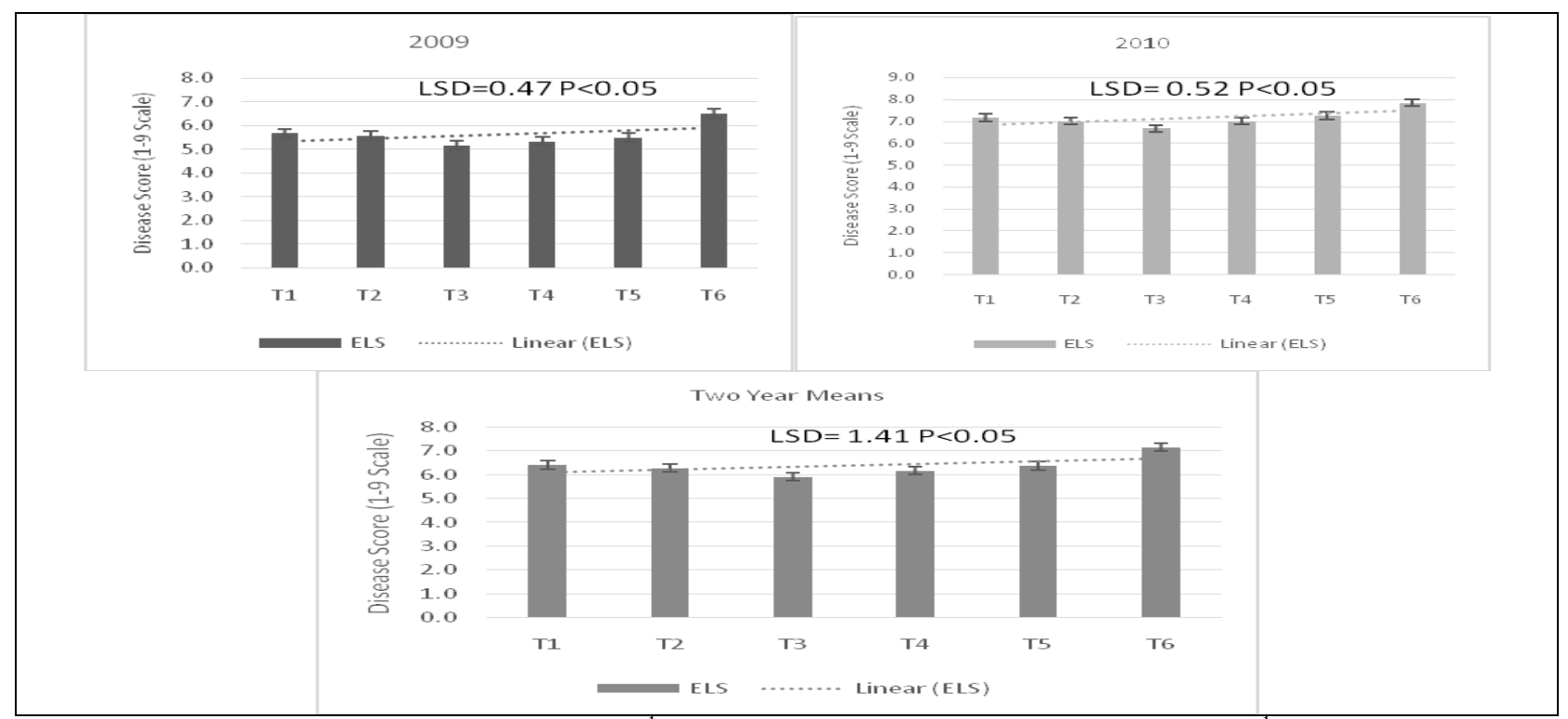

$\mathrm{T}_{1}$ : Seed treatment with T. harzianum $\left(10 \mathrm{~g} \mathrm{~kg}^{-1}\right.$ seed $)$ and two sprays of hexaconazole $\left(1 \mathrm{ml} \mathrm{l}^{-1}\right), \mathrm{T}_{2}$ : Seed treatment with mancozeb $\left(3 \mathrm{~g} \mathrm{~kg}^{-1}\right)$ and two spray of hexaconazole $\left(1 \mathrm{ml} \mathrm{l}^{-1}\right), \mathrm{T}_{3}$ : Seed treatment with tebuconazole $\left(1.5 \mathrm{~g} \mathrm{~kg}^{-1}\right.$ seed) and two foliar spray of tebuconazole $\left(1 \mathrm{ml} \mathrm{l}^{-1}\right), \mathrm{T}_{4}$ : Soil application of T. harzianum $\left(4.0 \mathrm{~kg} \mathrm{ha}^{-1}\right)$ and castor cake $\left(250 \mathrm{~kg} \mathrm{ha}^{-1}\right)$ and two spray of hexaconazole $\left(1 \mathrm{ml} \mathrm{l}^{-1}\right), \mathrm{T}_{5}$ : Seed treatment with $T$. harzianum $\left(10 \mathrm{~g} \mathrm{~kg}^{-1}\right)+\mathrm{T}_{4}$; $\mathrm{T}_{6}$ : Control (without any seed treatment, only water spray) 
Fig.6 Effect of different treatments on late leaf spot (LLS) severity. Least significant difference (LSD) values were calculated at $P<0.05$

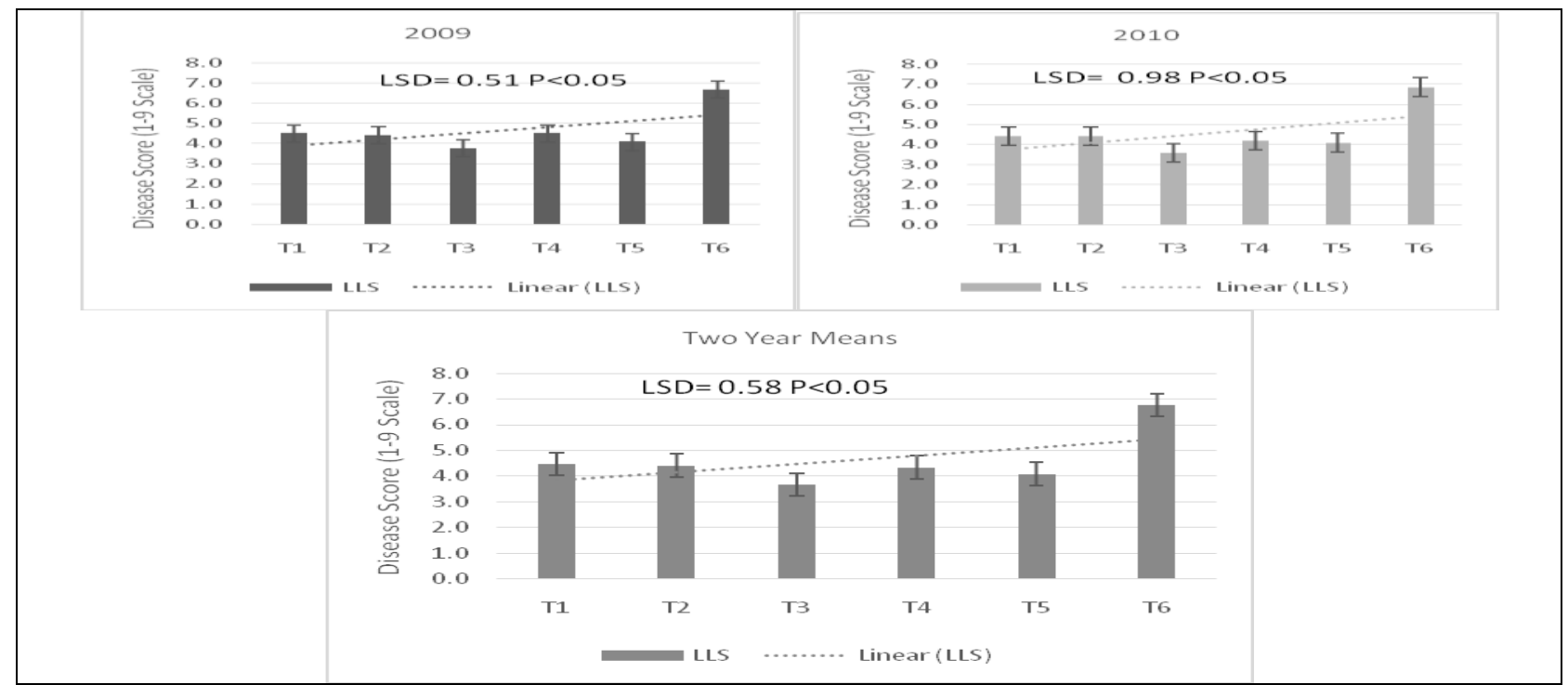

$\mathrm{T}_{1}$ : Seed treatment with $T$. harzianum $\left(10 \mathrm{~g} \mathrm{~kg}^{-1}\right.$ seed) and two sprays of hexaconazole $\left(1 \mathrm{ml} \mathrm{l}^{-1}\right), \mathrm{T}_{2}$ : Seed treatment with mancozeb $\left(3 \mathrm{~g} \mathrm{~kg}^{-1}\right)$ and two spray of hexaconazole $\left(1 \mathrm{ml} \mathrm{l}^{-1}\right), \mathrm{T}_{3}$ : Seed treatment with tebuconazole $\left(1.5 \mathrm{~g} \mathrm{~kg}^{-1}\right.$ seed) and two foliar spray of tebuconazole $\left(1 \mathrm{ml} \mathrm{l}^{-1}\right), \mathrm{T}_{4}$ : Soil application of T. harzianum $\left(4.0 \mathrm{~kg} \mathrm{ha}^{-1}\right)$ and castor cake $\left(250 \mathrm{~kg} \mathrm{ha}^{-1}\right)$ and two spray of hexaconazole $\left(1 \mathrm{ml} \mathrm{l}^{-1}\right), \mathrm{T}_{5}$ : Seed treatment with $T$. harzianum $\left(10 \mathrm{~g} \mathrm{~kg}^{-1}\right)+\mathrm{T}_{4}$; $\mathrm{T}_{6}$ : Control (without any seed treatment, only water spray)

Fig.7 Effect of different treatments on rust severity. Least significant difference (LSD) values were calculated at $P<0.05$

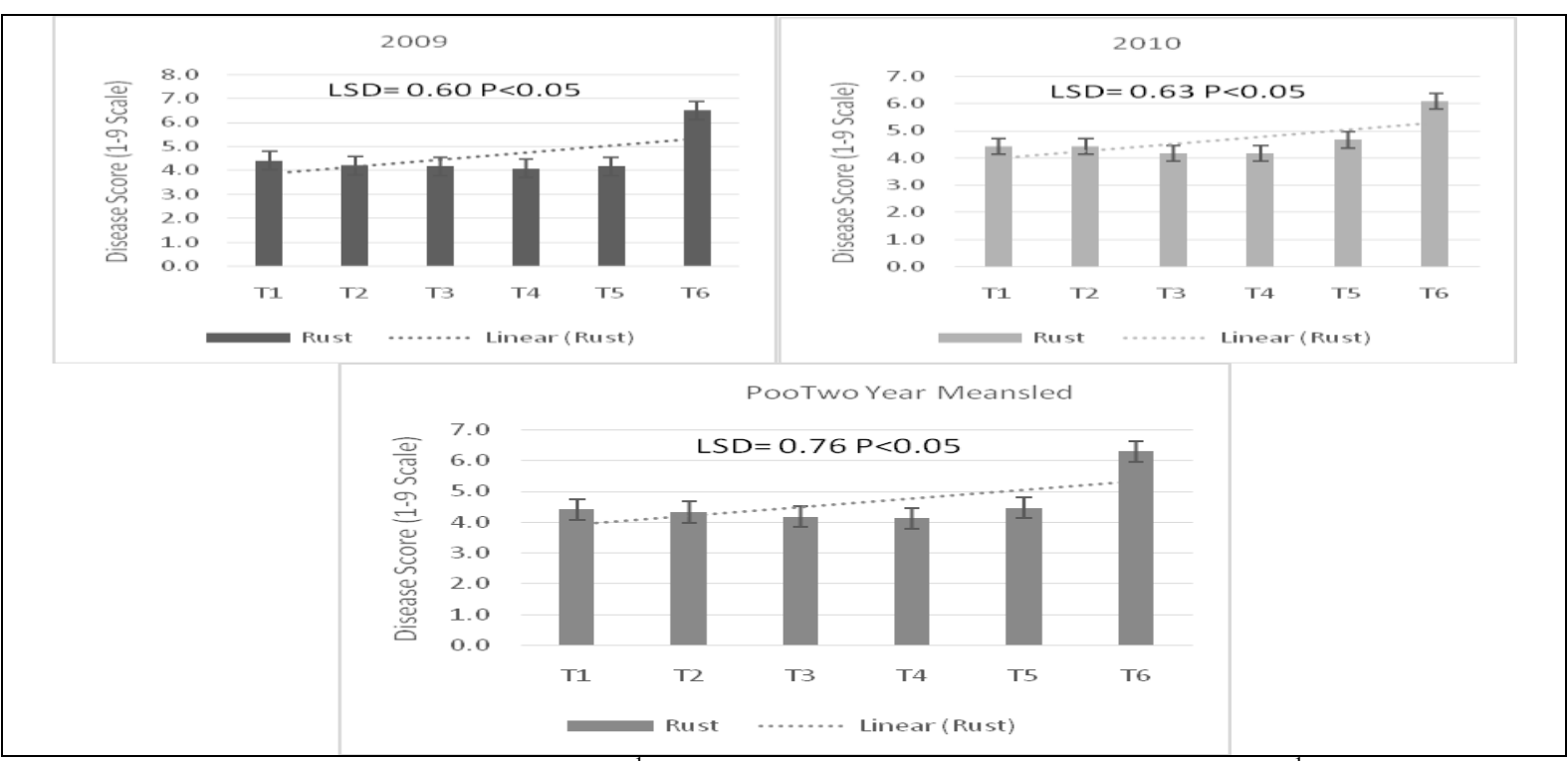

$\mathrm{T}_{1}$ : Seed treatment with $T$. harzianum $\left(10 \mathrm{~g} \mathrm{~kg}^{-1}\right.$ seed) and two sprays of hexaconazole $\left(1 \mathrm{ml} \mathrm{l}^{-1}\right), \mathrm{T}_{2}$ : Seed treatment with mancozeb $\left(3 \mathrm{~g} \mathrm{~kg}^{-1}\right)$ and two spray of hexaconazole $\left(1 \mathrm{ml} \mathrm{l}^{-1}\right), \mathrm{T}_{3}$ : Seed treatment with tebuconazole $\left(1.5 \mathrm{~g} \mathrm{~kg}^{-1}\right.$ seed) and two foliar spray of tebuconazole $\left(1 \mathrm{ml} \mathrm{l}^{-1}\right), \mathrm{T}_{4}$ : Soil application of $T$. harzianum $\left(4.0 \mathrm{~kg} \mathrm{ha}^{-1}\right)$ and castor cake $\left(250 \mathrm{~kg} \mathrm{ha}^{-1}\right)$ and two spray of hexaconazole $\left(1 \mathrm{ml} \mathrm{l}{ }^{-1}\right), \mathrm{T}_{5}$ : Seed treatment with $T$. harzianum $\left(10 \mathrm{~g} \mathrm{~kg}^{-1}\right)+\mathrm{T}_{4}$; $\mathrm{T}_{6}$ : Control (without any seed treatment, only water spray) 
Fig.8 Effect of different treatments on pod yield $\left(\mathrm{kg} \mathrm{ha}^{-1}\right)$ and haulm yield $\left(\mathrm{kg} \mathrm{ha}^{-1}\right)$ of Peanut. Least significant difference (LSD) values were calculated at $P<0.05$

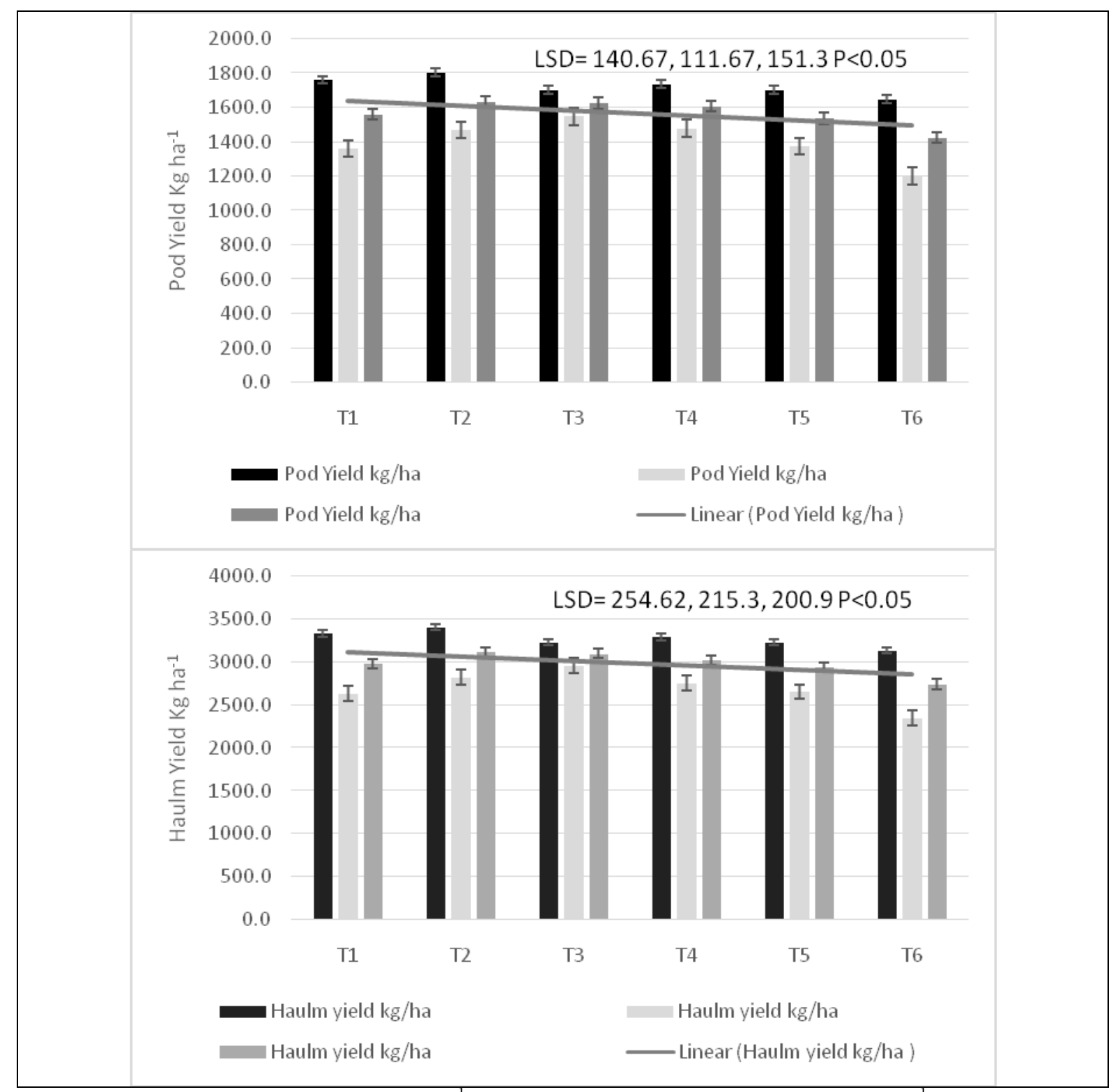

$\mathrm{T}_{1}$ : Seed treatment with $T$. harzianum $\left(10 \mathrm{~g} \mathrm{~kg}^{-1}\right.$ seed) and two sprays of hexaconazole $\left(1 \mathrm{ml} \mathrm{l}^{-1}\right), \mathrm{T}_{2}$ : Seed treatment with mancozeb $\left(3 \mathrm{~g} \mathrm{~kg}^{-1}\right)$ and two spray of hexaconazole $\left(1 \mathrm{ml} \mathrm{l}^{-1}\right), \mathrm{T}_{3}$ : Seed treatment with tebuconazole $\left(1.5 \mathrm{~g} \mathrm{~kg}^{-1}\right.$ seed) and two foliar spray of tebuconazole $\left(1 \mathrm{ml} \mathrm{l}^{-1}\right), \mathrm{T}_{4}$ : Soil application of $T$. harzianum $\left(4.0 \mathrm{~kg} \mathrm{ha}^{-1}\right)$ and castor cake $\left(250 \mathrm{~kg} \mathrm{ha}^{-1}\right)$ and two spray of hexaconazole $\left(1 \mathrm{ml} \mathrm{l}^{-1}\right), \mathrm{T}_{5}$ : Seed treatment with $T$. harzianum $\left(10 \mathrm{~g} \mathrm{~kg}^{-1}\right)+\mathrm{T}_{4}$; $\mathrm{T}_{6}$ : Control (without any seed treatment, only water spray)

The results of the experiments indicated that all the treatments significantly reduced the incidence of soil-bornediseases and severity of foliar fungal diseases over the untreated control. Dandnaik et al., (2009) reported that hexaconazole as seed treatment showed the highest seed germination (75\%). Sharma and Gour (2009) noted that seed treatment with carbendazim and propiconazole also resulted in increased germination and seedling vigour of pea. Significantly higher germination percent (93) was observed with carbendazim 
as a seed treatment against Macrophomina phaseolina in cluster bean. Shrirao et al., (2009) reported that seed treatment with thiram + carbendazim + Trichoderma sp. effectively reduced the mortality $(75 \%)$ due to root and collar rot of soybean as compared to control. Similarly, seed treatments with carbendazim, captan, and carbendazim + captan were found effective and at par in recording significantly higher pre- and postemergence mortality due to collar rot in soybean (Pawar et al., 2009). Sreedevi et al., (2011) reported that the growth of peanut plants with the antagonist alone or in combination with pathogen was greater than in plants inoculated with pathogen alone. Sai et al., (2010), reported Trichoderma isolates TG2 was significantly superior over others in inhibiting the mycelial growth of $S$. rolfsii to the extent of $67.83 \%$. Harsukh et al., (2011) also reported that seed treatment with Trichoderma reduced the disease incidence in susceptible and tolerant varieties at 15 DAS, under A. niger infection.

In the present studies, the lowest incidence of soil-borne diseases was recorded during both the years of seed treatment with mancozeb and two sprays of hexaconazole and seed treatment with tebuconazole and two foliar sprays of tebuconazole in comparison to untreated control and significantly reduced the disease incidence. Johnson and Subramanyam (2010) and Dandnaik et al., (2009) also reported that seed treatment with hexaconazole showed the maximum reduction of stem rot incidence $(24 \%)$ in peanut. Minimum incidence of pre-emergence rot and post-emergence seedling rot (13\%) were recorded in treatment with carbendazim and thiophanete methyl compared to control (43 and 49 percent, respectively) in cluster bean (Jaiman et al., 2009). Gour and Sharma (2010) also reported that sprays of Folicur $250 \mathrm{EW}$ in the field, along with two standard controls, i.e. Tilt $25 \mathrm{EC}$ and contaf $5 \mathrm{EC}$ showed notable efficacy, resulting in low PDI of 8.7 and $16.4 \%$ and PEDC of 87.9 and $77.3 \%$, respectively, after 30 days. Tilt $25 \mathrm{EC}$ and Contaf 5 EC also showed disease control efficacy of 68.9 and $65.6 \%$, respectively. In the uninoculated control plots, the disease severity (PDI) was 28.5\%. All the concentrations of Folicur $250 \mathrm{EW}$ were significantly superior in PEDC to the standard controls, Tilt 25 EC (68.9\%) and contaf 5 EC (65.6\%). Augusto and Brenneman (2012) studied the movement of systemic fungicides in three terminal, fully expanded leaves of primary lateral branches of 'Tifrunner' peanut treated with prothioconazole + tebuconazole (Provost, $0.29 \mathrm{~kg}$ a.i./ha), azoxystrobin (Abound, $0.31 \mathrm{~kg}$ a.i./ha), or flutolanil (moncut, $0.79 \mathrm{~kg}$ a.i./ha) in field experiments. Their results demonstrated acropetal protection by all the fungicides evaluated and indicated that prothioconazole + tebuconazole or prothioconazole applied to foliage can sometimes reduce diseases in the lower, nontreated portions of the plant.

The highest PEDC (per cent efficiency over disease control) was observed in $\mathrm{T}_{2}$ and $\mathrm{T}_{3}$ for soil borne diseases in comparison to other treatments applied. These results were also in concurrence with Devi and Prasad (2009) who reported that combined effect of seed treatment with $T$. viride and captan resulted in significant reduction of collar rot and combination of antagonist and fungicide also improved the growth parameters like the length of the plant, biomass and yield besides decreasing the disease incidence. Muhammad and Bdliya (2011) evaluated the effects of variety and fungicide (mancozeb) on Cercospora leaf spot disease of peanut and found that disease incidence and disease severity were significantly higher in 'ExDakar' variety and untreated seeds, thus had lower yields. Higher seed and haulm yield were recorded by RRB, ICGV-86024, as well as 2 and $4 \mathrm{~kg} \mathrm{ha}^{-1}$ as a result of lower disease 
incidence and severity. Wann et al., (2011) also evaluated the efficacy of three approved fungicides for leaf spot control on peanut under organic management. Copper sulfate+ Bacillus subtilis reduced leaf spot defoliation compared to the control. Yet, all three fungicides improved yields under heavy leaf spot pressure. Combining high-yielding, disease-resistant cultivars and organically approved fungicides can significantly reduce leaf spot and improve yield potential of peanut under organic management. Augusto and Brenneman (2011) reported that night and day applications of a systemic fungicide for leaf spot disease control were similar across post-spray irrigation, but pyraclostrobin and prothioconazole + tebuconazole had the lowest disease score. Interaction of fungicide, application timing, and post-spray irrigation was significant for stem rot and yield. Night application of prothioconazole + tebuconazole, flutolanil + propiconazole or pyraclostrobin showed the most increase in stem rot control and yield compared with day application among the evaluated fungicides, but the positive effects on stem rot control and yield were minimal with post-spray irrigation.

The observation on foliar diseases revealed that all the treatments had significantly reduced PDI (per cent disease incidence) in comparison to untreated control. Seed treatment with tebuconazole and two foliar sprays of tebuconazole was found most effective among all the treatments. The lowest PDI of foliar diseases were recorded in $T_{3}$ whereas it was recorded highest in the untreated control. The PEDC (per cent efficacy over disease control) was also very high in $\mathrm{T}_{3}$ in comparison to other treatments. Hagan et al., (2010) also reported azoxystrobin, chlorothalonil, and tebuconazole, for the control of ELS and stem rot and found the incidence of these diseases were lower with the application of azoxystrobin than chlorothalonil in the 2 out of 3 year's programs. The azoxystrobin program significantly increased yield compared with the chlorothalonil or tebuconazole. The yield was also higher in the tebuconazole treatment compared with chlorothalonil. Grichar et al., (2010) conducted a field experiment to determine both foliar and soil-borne disease control and peanut response to prothioconazole + tebuconazole (Provost) fungicide treatments when compared with other fungicides commonly used in peanut in different peanut growing areas of Texas. ELS control with prothioconazole + tebuconazole was comparable with azoxystrobin (Abound), chlorothalonil (Bravo), or tebuconazole (Folicur) and incidence of the disease was reduced at least $36 \%$ relative to the untreated check. Southern stem rot control with prothioconazole + tebuconazole was comparable to azoxystrobin or pyraclostrobin (Headline) and all fungicides reduced disease incidence at least $60 \%$ when compared to the untreated check. Peanut yields with prothioconazole + tebuconazole were increased at least $85 \%$ over the untreated check when ELS was present and at least 34\% over those of the untreated check when Southern stem rot was present.

The data on biological yield (pod and haulm) of peanut showed the significant effect of treatments on the enhancement of the yield over the untreated control. The highest pod yield was recorded in $T_{2}$ and $T_{3}$ whereas it was lowest in the untreated control. The haulm yield was also found highest in $\mathrm{T}_{2}$ and $\mathrm{T}_{3}$ in comparison to control. Both the treatment $T_{2}$ and $T_{3}$ showed the highest PIDC (per cent increase over disease control) for pod yield and for haulm yield, respectively, among all the treatments. The results confirmed the findings of Johnson and Subramanyam (2010) and Dandnaik et al., (2009) who reported that seed treatment with 
hexaconazole gave higher pod yield of 1400 $\mathrm{kg} / \mathrm{ha}$ in peanut. The effect of seed treatment in increasing the yields was reported by several workers viz., De et al., (2003) with carbendazim + thiram against wilt of linseed, Ramkishore and Singh (2008) with carbendazim against wilt of linseed, Jaiman and Jain (2008) with carbendazim against blight and root rot of cluster bean, Dudi and Lodha (2003) with carbendazim against seedling diseases of peanut and Dutta and Das (2002) with mancozeb against collar rot of tomato. Gour and Sharma (2010) also reported that for pod yield, there was no significant difference in the two concentrations of Folicur $250 \mathrm{EW}$ (2550 and $2510 \mathrm{~kg} / \mathrm{ha}$ at 187.50 and $156.25 \mathrm{~g}$ a.i./ ha, respectively compared to $1720 \mathrm{~kg} / \mathrm{ha}$ in the inoculated control). The highest percentage increase of pod $(48.2 \%)$ was observed in Folicur $250 \mathrm{EW}$ at $187.5 \mathrm{~g}$ a.i./ha, followed by at $156.25 \mathrm{~g}$ a.i./ha (45.9\%). In applications of Tilt $25 \mathrm{EC}$ and Contaf $5 \mathrm{EC}$, the percentage increase in pod yield was 36.0 and $35.5 \%$, respectively compared to the inoculated control. The data showed that the cost of treatment are very important to manage the disease so it is suggested that treatment $\mathrm{T}_{2^{-}}$ seed treatment with mancozeb $\left(3 \mathrm{~g} \mathrm{~kg}^{-1}\right)$ and two sprays of hexaconazole $\left(1 \mathrm{ml} \mathrm{l}^{-1}\right)$ will be most suitable for management of peanut disease.

\section{Acknowledgements}

This research was funded by the ICARDirectorate of Groundnut Research, Junagadh, Gujarat, India. The authors express sincere gratitude to the Director, ICAR-DGR for his constant support and encouragement during this research programme.

\section{References}

Augusto J, and Brenneman TB. 2012. Assessing systemicity of peanut fungicides through bioassay of plant tissues with Sclerotium rolfsii. Plant Disease. 96(3): 330-337.

Augusto J, Brenneman TB, 2011. Implications of fungicide application timing and post-spray irrigation on disease control and peanut yield. Peanut Science. 38: 48-56.

Beasley, J., Baldwin, J., and Padgett, B. 1997. Peanut Production Field Guide. Univ. of Ga. Coop. Ext. Serv. College of Agric. and Env. Sci. Bulletin 1146. 86 pp.

Dandnaik B P, Patil D T, Chavanand M H and Dandnaik A B 2009. Chemical control of stem rot of groundnut caused by Sclerotium rolfsii. Journal of Mycology and Plant Pathology 39: 185.

De R K, Dwivedi R P and UditNarain 2003. Biological control of lentil wilt caused by Fusarium oxysporum f.sp. lentis. Annals of Plant Protection Sciences 11: 46-52.

Devi MC and Prasad RD 2009. Biointensive management of collar rot of groundnut caused by Aspergillus niger. Journal of Biological Control 23: 21-24.

FAO, 2013. http://faostat3.fao.org/browse/ Q/QC/E. Accessed 28 September 2015.

Gour HN, and Pankaj Sharma. 2010. Evaluation of fungicides in vitro and in vivo against Sclerotium rolfsii Sacc. causing root rot of groundnut. Indian Phytopathology. 63(3): 352-353.

Grichar WJ, Jaks AJ and Woodward J 2010.Using prothioconazole plus tebuconazole for foliar and soil-borne disease control in Texas peanut. Crop Management. (April): CM-2010-040502-RS.

Hagan AK, Campbell HL, Bowen KL, Wells L and Goodman R 2010. Managing early leaf spot and stem rot with reduced fungicide inputs on diseaseresistant peanut cultivars. Peanut Science. 37: 129-136. 
HarsukhGajera, KaluRakholiya, and Dinesh Vakharia. 2011 Bioefficacy of Trichoderma isolates against Aspergillus niger Van Tieghem inciting collar rot in groundnut (Arachis hypogaea L.). Journal of Plant Protection Research. 51(3): 240-247.

DGR, 2014. Annual report of All India Coordinated Research Project on Groundnut 2014-15 (kharif, p1-123 and rabi pp 43), ICAR-Directorate of Groundnut Research, Junagadh, Gujarat, India.

Jaiman R K, Jain S C, and Pankaj Sharma 2009. Field evaluation of fungicides, bioagents and soil amendments against root rot caused by Macrophomina phaseolina in cluster bean. Journal of Mycology and Plant Pathology 39: 74 76

DudiJ R and Lodha P C 2003. Compatibility of seed dressing fungicides with rhizobium in groundnut. National seminar: stress management in oil seeds. Jan 28-30, 2003. pp 24-25.

Johnson M, and Subramanyam K 2010. Evaluation of different fungicides against seed and soil borne diseases of groundnut. Indian Journal of Plant Protection 38: 80-83.

GOI, 2014. Statistical Appendix: Economic Survey 2014-15, Ministry of Finance, Government of India, p 148.http://indiabudget.nic.in/es2014-15/ estat1.pdf. Accessed 28 September 2015.

Moss, J. P., and Rao, R. R. 1995. The peanut

- reproductive development to plant maturity. In Advances in Peanut Science. pp. 1-13. American Peanut Research and Education Society, Inc. Stillwater, Oklahoma.

Muhammad AS and Bdliya BS 2011. Effects of variety and fungicidal rate on Cercospora leaf spots disease of Peanut in the Sudan Savanna. Nigerian Journal of Basic and Applied Sciences. 19: 135141.

Onobri A. 2007. Routine statistical analysis of field experiments by using an excel extension. Proceedings 6th national conference Italian Biometric Society: "La statisticanelle science dellavita e dell anb I ante" Pisa, 20-22 June, pp. 93-96.

Panse V G and Sukhatme P V 1978. Statistical methods for Agricultural Workers. Indian Council of Agricultural Research, New Delhi. 136 pp.

Pawar A K, Suryawanshi A P, Magar S J, Badgire D R and Devgir S S. 2009. Management of soybean collar rot caused by Sclerotium rolfsii Sacc. Journal of Mycology and Plant Pathology 39: 187.

PranbDutta and Das B C 2002. Management of collar rot of tomato by Trichoderma spp. and chemicals. Indian Phytopathology 55: 235-237.

Rakesh Kumar Jaiman and Jain S C 2008. Macrophomina phaseolina in cluster bean (Cyamopsis tetragonoloba) seeds and its control. Journal of Mycology and Plant Pathology 38: 403-404.

Ram Kishore and Jyoti Singh 2008 Evaluation of fungicides against Fusarium oxysporum f.sp. lentis of linseed. Annals of Plant Protection Sciences 16: 165-167.

Sai LV, Anuradha P, Vijayalakshmi K, and Reddy NPE. 2010. Biocontrol of stem rot of groundnut incited by Sclerotium rolfsii and in vitro compatibility of potential native antagonists with fungicides. Journal of Pure and Applied Microbiology. 4(2): 565-570.

Sharma Pankaj and Gour H N 2009.Location of seed borne mycoflora in pea (Pisum sativum L.) and efficacy of fungicides on germination and seedling vigour. Journal of Mycology and Plant Pathology 39: 90-93. 
ShriraoA V, Gawade D B, Shrirao R A, Dikey H H and Patil S P 2009. Efficacy of fungicides and bioagents against the control of root or collar rot of soybean. Journal of Mycology and Plant Pathology 39: 184.

Singh RS,1998. NBSS Technical Bulletin "Soil Resource Appraisal Research Farm, NRCG, Junagadh" No. 2810. NBSS\&LUP regional station, Udaipur. Pp 1-57.

Sreedevi B, Devi MC, and Saigopal DVR. 2011. Isolation and screening of effective Trichoderma spp. against the root rot pathogen Macrophomina phaseolina. International Journal of Agricultural Technology. 7(3): 623-635.

Subrahmanyam P, McDonald D, Waliyar E, Reddy LJ, Nigam SN, Gibbons RW, Ramanatha Rao V, Singh AK, Pande S,
Reddy PM, and Subba Rao PV 1995. Screening methods and sources of resistance to rust and late leaf spot of groundnut (In En. Summaries in En, Fr, Sp, and Pt.). Information Bulletin no. 47. Patancheru 502 324, Andhra Pradesh, India: International Crops Research Institute for the Semi-Arid Tropics. 24 pp.

Thind T S 2008. Fungicidal resistance: a perpetual challenge in disease control. Journal of Mycology and Plant Pathology 38: 407-418.

Wann DQ, Tubbs RS and Culbreath AK 2011. Genotype and approved fungicide evaluation for reducing leaf spot diseases in organically-managed peanut. Plant Health Progress. (October): PHP2010-1027-01-RS.

\section{How to cite this article:}

Jadon, K.S., P.P. Thirumalaisamy, Vinod Kumar, V.G. Koradia and Padavi, R.D. 2017. Integrated Management of Major Foliar and Soil-Borne Diseases of Peanut (Arachis hypogaea L.) with Fungicides, Trichoderma and Castor Cake. Int.J.Curr.Microbiol.App.Sci. 6(12): 18841899. doi: https://doi.org/10.20546/ijcmas.2017.612.215 